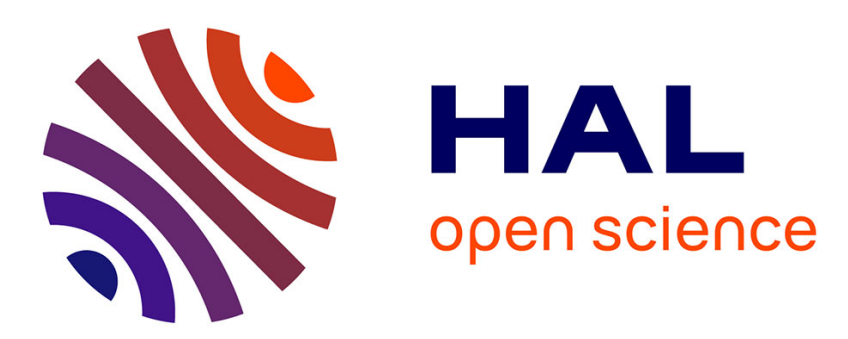

\title{
Un contrat à obligation d'exécution peut-il résoudre le problème du hold-up?
}

M'hand Fares

\section{To cite this version:}

M'hand Fares. Un contrat à obligation d'exécution peut-il résoudre le problème du hold-up?. Revue Economique, 2011, 62 (3), pp.545-556. 10.3917/reco.623.0545 . hal-02647357

\section{HAL Id: hal-02647357 https://hal.inrae.fr/hal-02647357}

Submitted on 29 May 2020

HAL is a multi-disciplinary open access archive for the deposit and dissemination of scientific research documents, whether they are published or not. The documents may come from teaching and research institutions in France or abroad, or from public or private research centers.
L'archive ouverte pluridisciplinaire HAL, est destinée au dépôt et à la diffusion de documents scientifiques de niveau recherche, publiés ou non, émanant des établissements d'enseignement et de recherche français ou étrangers, des laboratoires publics ou privés. 


\title{
Un contrat à obligation d'exécution peut-il résoudre le problème du hold-up ?
}

\author{
M'hand Fares*
}

\begin{abstract}
Cet article cherche à déterminer les conditions sous lesquelles un contrat à obligation d'exécution peut résoudre le problème classique du hold-up. Nous mettons en évidence deux résultats. D'une part, dans un environnement très général, la solution efficace ne peut être obtenue que si le contrat contrôle aussi le processus de renégociation. D'autre part, dans un environnement spécifique où les fonctions de bénéfice et de coût satisfont une condition de séparabilité, la solution efficace est implémentée parce qu'il existe une équivalence entre cette condition et l'hypothèse d'indépendance à l'état du monde. Dans ce cas, le contrat d'obligation d'exécution n'est pas nécessaire car un contrat volontaire est tout aussi efficace.
\end{abstract}

\section{CAN A SPECIFIC PERFORMANCE \\ CONTRACT SOLVE THE HOLD-UP PROBLEM?}

This paper aims to define the conditions under which a specific performance contract can solve the hold-up problem. We mainly show two results. First, in a very general environment the efficient solution is achieved only with renegotiation design. Second, in a specific environment where the valuation functions satisfy a separability condition, the efficient solution is implemented only because there is an equivalence result between this condition and a state independence assumption. This implies that a specific performance contrat is unecessary since a volontary contract is also able to achieve efficiency.

Classification JEL : D23, D86, K12, L14

\section{INTRODUCTION}

Contrairement à l'univers walrasien où les échanges sont impersonnels et les contrats signés contingents à l'ensemble des états du monde, la pratique contractuelle met en évidence des situations où les parties développent des investissements idiosyncratiques et signent des contrats incomplets (Williamson

* INRA (UMR 1248, AGIR) et Université Paris 1. Correspondance : BP 52627, F-31326 CastanetTolosan Cedex France. Courriel : mfares@univ-paris1.fr

Je remercie les rapporteurs anonymes, Tore Ellingsen, David Martimort, Anne Perrot, et Pierre Picard pour leurs commentaires. 
[1985], Hart [1995]). Cette incomplétude, liée à l'incapacité des parties à inclure tous les états du monde susceptibles de survenir dans la relation, peut être due soit à un problème de description de ces états (Dye [1985], Anderlini et Felli [1994], Battigali et Maggi [2002], Al Najjar et al. [2006]), soit à un problème de vérifiabilité de ces états par une tierce partie (Hart et Moore [1988], Anderlini et al. [2007]). Lorsque l'état du monde est réalisé, les parties peuvent compléter le contrat initial à travers une procédure de renégociation. Si la renégociation parvient à assurer un niveau d'échange efficace en symétrie d'information (efficacité ex post), le partage du surplus qui résulte de cette renégociation génère cependant des distorsions en terme d'incitation à investir (inefficacité ex ante). Ce résultat de sous-investissement est communément appelé « le problème du hold-up » (Klein et al. [1978], Williamson [1985]).

La théorie des contrats incomplets avance deux types de solution à ce problème. Premièrement, la solution des droits de propriété (Grossman et Hart [1986], Hart [1995]). En allouant à l'une des parties des droits résiduels de contrôle sur des actifs physiques engagés dans la relation, on augmente son pouvoir de marchandage dans la renégociation, et donc ses incitations à investir. Cependant, cette solution ne réduit que partiellement le problème du hold-up. En effet, le caractère exclusif des droits de propriété sur les actifs physiques implique qu'allouer plus de droits résiduels à l'une des parties revient à en donner moins à son partenaire. Ce qui réduit d'autant le pouvoir de marchandage de ce dernier, et donc ses incitations à investir. Deuxièmement, la solution du contrat de long terme (Hart et Moore [1988], MacLeod et Malcomson [1993], Aghion et al. [1994], Nöldeke et Schmidt [1995]). L'article pionnier de Hart et Moore [1988] met en évidence un résultat négatif : un contrat volontaire de long terme ne peut mettre en œuvre la solution efficace car la partie qui n'a aucun pouvoir de marchandage ne reçoit rien de plus que le gain en absence d'échange ; gain qui est indépendant de son investissement. Aghion et al. [1994] montrent que ce résultat négatif peut être dépassé si l'on considère une classe de contrats plus large que celle des contrats volontaires. La solution efficace peut être atteinte si le contrat initial est à obligation d'exécution (specific performance), et s'il permet de contrôler l'allocation du pouvoir de marchandage dans le processus de renégociation. Plus précisément, ce contrat devra être tel : (i) que le niveau d'échange minimum permette à la partie sans pouvoir de marchandage d'obtenir des incitations correcte à investir et non un simple gain d'absence d'échange ; (ii) qu'il assure au partenaire d'avoir l'ensemble du pouvoir de marchandage capable de lui fournir les bonnes incitations à investir. Edlin et Reichelstein [1996] suggèrent cependant que la solution efficace peut être atteinte sans faire l'hypothèse que le contrat initial contrôle le processus de renégociation (condition (ii)). Un simple contrat à obligation d'exécution (condition (i)) serait suffisant si les fonctions de bénéfice et de coût sont additivement séparables.

Notre article analyse les conditions sous lesquelles un simple contrat à obligation d'exécution peut résoudre le problème du hold-up. Dans un premier temps, nous mettons en évidence le résultat négatif suivant : dans un environnement très général, un simple contrat à obligation d'exécution ne peut mettre en œuvre le niveau d'investissements de premier rang (proposition 1). Une structure supplémentaire est nécessaire. La solution efficace requiert, en effet, de spécifier deux niveaux d'échange différents; différents car tous deux supérieurs au niveau moyen d'échange attendu. Un tel biais «vers le haut» des quantités initiales tient au fait que les fonctions de bénéfice et de coût sont directement dépendantes 
de l'aléa. Mais ce type de contrat à obligation d'exécution avec deux niveaux d'échange ne peut être mis en œuvre par un juge. Ce dernier ne pouvant déterminer qui des deux protagonistes a rompu le contrat, il ne sait quelle quantité faire exécuter. Nous montrons alors que seul un contrat à obligation d'exécution, contrôlant aussi le processus de marchandage (condition (ii)), permet de ne spécifier qu'une seule quantité de défaut. Le contrat, ainsi rendu exécutable, peut être paramétré afin de répliquer la solution de premier rang. Dans un second temps, nous montrons que, dans l'environnement spécifique considéré par Edlin et Reichelstein [1996], le recours au contrat à obligation d'exécution n'est pas nécessaire. $\mathrm{Si}$, dans un tel environnement, un contrat à obligation d'exécution peut être efficace sans contrôle du processus de renégociation, c'est parce que la condition de séparabilité d'Edlin et Reichelstein [1996] est équivalente à une hypothèse d'indépendance à l'aléa (proposition 2). Cette hypothèse d'indépendance implique en effet que le biais sur les quantités, mis en lumière précédemment, disparaît. Un contrat à obligation d'exécution stipulant une quantité unique peut alors mettre en œuvre la solution de premier rang. Cependant, sous cette hypothèse, un tel contrat n'est plus nécessaire, puisqu'un contrat volontaire met en œuvre de façon triviale la solution efficace (proposition 3).

En montrant qu'un simple contrat à obligation d'exécution n'est pertinent et efficace que sous des conditions restrictives, notre travail permet d'expliquer la faible utilisation de ce remède à la rupture des contrats que constitue l'obligation d'exécution (Lando et Rose [2004]). En outre, notre résultat, selon lequel ce type de contrat n'est efficace que s'il contrôle aussi le processus de renégociation, rend compte des rares cas d'utilisation avérée de ce remède. En effet, comme le montre Lando et Rose [2004] et Shavell [2006] les juges ne mettent en œuvre l'obligation d'exécution que lorsqu'ils sont confrontés à des contrats " complexes » (comme les contrats de construction) capables de définir et de contrôler les conditions de renégociation et/ou de rupture de la relation. Ceci suggère que la structuration des contrats importe dans l'efficacité des remèdes à la rupture des contrats.

Le reste de l'article est organisé de la façon suivante. Dans la section 2, nous présentons le cadre d'analyse et nous étudions l'efficacité du contrat à obligation d'exécution, puis celle du contrat volontaire. La section 3 apporte quelques éléments de conclusion.

\section{Le modèle}

Un vendeur et un acheteur s'engagent dans une relation verticale de long terme. Ils écrivent un contrat qui spécifie la livraison, à une date précise dans le futur, d'une quantité donnée $q \in \mathcal{R}_{+}$du bien produit par le vendeur, en contrepartie d'un transfert $p \in \mathcal{R}$. Le coût de production et le bénéfice sont supposés stochastiques et affectés par la réalisation d'un variable aléatoire $\omega \in \Omega$ (dont la distribution suit une fonction connue $F(\omega)$ ) et par les niveaux d'investissements des parties $(\sigma \in[0, \infty)$ pour le vendeur, et $\beta \in[0, \infty)$ pour l'acheteur). Ces investissements sont mis en place avant la réalisation de $\omega$, et sont spécifiques à la relation. C'est-à-dire que les parties ne peuvent récupérer les coûts de mise en place de ces investissements s'ils quittent la relation. Soit $\mathrm{C}(q, \omega, \sigma)$ le coût de production du vendeur qui s'engage à livrer $q$ unités de bien, étant donné l'aléa $\omega$ et le niveau d'investissement $\sigma$. De même, soit $\mathrm{V}(q, \omega, \beta)$ le 
bénéfice (brut) que réalise l'acheteur avec $q$ unités du bien, étant donné $\omega$ et $\beta$. Nous faisons l'hypothèse que $\mathrm{V}(q, \omega, \beta) \mathrm{C}(q, \omega, \sigma)$ sont des fonctions croissantes en $q$ et $\mathrm{V}(q, \omega, \beta)-\mathrm{C}(q, \omega, \sigma)$ est (strictement) croissant en $(\beta, \sigma)$ à un taux (strictement) décroissant. Le cas économiquement intéressant est celui où les incitations à investir sont sensibles au niveau d'échange attendu, c'est-à-dire que $\mathrm{V}_{\beta q}>0$ et $\mathrm{C}_{\sigma q}<0$. Supposons aussi qu'un meilleur aléa $\omega$ augmente (diminue) le bénéfice marginal (coût de production marginal) de l'investissement, i.e. $\mathrm{V}_{\beta \omega}>0\left(\mathrm{C}_{\sigma \omega}<0\right)$. En outre, le bénéfice et le coût en absence d'échange sont supposés nuls, c'est-à-dire que pour tout $\omega, \beta, \sigma: \mathrm{V}(0, \omega, \beta)=\mathrm{C}(0, \omega, \sigma)=0$. Cette hypothèse formalise simplement l'idée de la spécificité des investissements, puisque leur coût ne peut être récupéré en cas d'absence d'échange entre les partenaires. Enfin, dans la dérivation de nos résultats nous faisons l'hypothèse simplificatrice suivante

HYPOTHÈSE $1 . \quad \mathrm{V}(q, \omega, \beta)=v_{1}(\omega, \beta) q+v_{2}(\omega, \beta)$ et

$$
\mathrm{C}(q, \omega, \sigma)=c_{1}(\omega, \sigma) q+c_{2}(\omega, \sigma) \text {. }
$$

L'hypothèse 1 dit simplement que les fonctions de bénéfice et de coût sont linéaires en $q$. Ce qui implique que ces fonctions augmentent uniformément pour des niveaux de $(\omega, \beta)$ plus élevés. Cette hypothèse est faite tout au long de l'article.

La séquence des événements de la relation contractuelle entre le vendeur et l'acheteur est la suivante. À la date 0 , les parties s'engagent sur un contrat de long terme non contingent. À la date 1, elles décident simultanément de leur niveau d'investissement. À la date 2, le vendeur et l'acheteur observent l'aléa ainsi que les niveaux d'investissement réalisés. L'échange ou la rupture puis la livraison et le paiement interviennent à la date 3 , après quoi toute requête auprès du juge est examinée en cas de rupture. Entre les dates 2 et 3, une renégociation du contrat initial peut avoir lieu si les parties le souhaitent.

\section{La solution de premier rang}

Si les deux parties sont neutres au risque, étant donné la réalisation d'un aléa $\omega$ et les choix d'investissements $(\beta, \sigma)$, leurs préférences sont décrites par les fonctions d'utilité suivantes : $u_{\mathrm{B}}=\left[q v_{1}(\omega, \beta)+v_{2}(\omega, \beta)\right]-p$ pour l'acheteur et $u_{\mathrm{S}}=p-\left[q c_{1}(\omega, \sigma)+c_{2}(\omega, \sigma)\right]$ pour le vendeur. Le surplus social de la relation est alors défini par

$$
\phi(q, \omega, \beta, \sigma) \equiv u_{\mathrm{B}}+u_{\mathrm{S}}=q\left[v_{1}(\omega, \beta)-c_{1}(\omega, \sigma)\right]+\left[v_{2}(\omega, \beta)-c_{2}(\omega, \sigma)\right] .
$$

Pour tout $(\beta, \sigma)$, soit

$$
\left\{q^{*}(\omega, \beta, \sigma), p^{*}(\omega, \beta, \sigma): \omega \in \Omega\right\}
$$

l'échange de premier rang contingent à $(\beta, \sigma)$, avec

$$
q^{*}(\omega, \beta, \sigma)=\underset{q}{\operatorname{argmax}} \phi(q, \omega, \beta, \sigma)
$$

la quantité qui maximise le surplus social ex post. Les investissements de premier rang $\left(\beta^{*}, \sigma^{*}\right)$ maximisent le surplus social net attendu

$$
\left(\beta^{*}, \sigma^{*}\right) \equiv \underset{\beta, \sigma}{\operatorname{argmax}} \int \phi\left(q^{*}(\omega, \beta, \sigma), \omega, \beta, \sigma\right) d \mathrm{~F}(\omega)-\beta-\sigma
$$


ce surplus social net attendu est la différence entre le gain total attendu et le coût direct de mise en place des investissements.

La solution de premier rang, qui assure à la fois l'efficacité ex post de l'échange et l'efficacité ex ante des niveaux d'investissement, peut être atteinte lorsque les variables $\beta, \sigma, \omega$ sont observables par les deux parties et vérifiables par un juge. En effet, lorsque les parties peuvent contracter sur le niveau d'échange et les niveaux d'investissements efficaces, un contrat complet stipulera que l'acheteur et le vendeur doivent mettre en place respectivement $\beta^{*}$ et $\sigma^{*}$ à la date 1 et échanger $q^{*}\left(\omega, \beta^{*}, \sigma^{*}\right)$ à la date 3 . Si l'une de ces obligations contractuelles n'est pas remplie, la partie qui a fait défaut devra payer une pénalité élevée à son partenaire à la date 3 .

\section{Les solutions atteignables avec un contrat à obligation d'exécution}

Suivant l'approche en terme de contrat incomplet, nous supposons que $\omega, \beta$, et $\sigma$ ainsi que $\mathrm{V}$ et $\mathrm{C}$ sont tous observables et non vérifiables par le juge. De ce fait, il est impossible d'écrire un contrat conditionnel sur ces variables : le contrat initial ne peut spécifier qu'une allocation non contingente $(\widetilde{q}, \widetilde{p})$. Ce contrat est supposé être à obligation d'exécution, c'est-à-dire que le juge peut imposer l'allocation non contingente si l'une des deux parties le demande. En terme légal, le juge utilise la règle de l'obligation d'exécution avec une possibilité de renégociation du contrat initial. Autrement dit, le juge met en œuvre le contrat initial sauf si les deux parties se mettent d'accord pour le renégocient et le remplacer par un autre contrat. S'il n'y a pas de rupture, nous supposons que la renégociation du contrat initial entre les dates 2 et 3 est telle que la quantité échangée correspond à la quantité efficace $q^{*}(\omega, \beta, \sigma)$ et les deux parties s'approprient le surplus net. Ce surplus net de la renégociation, noté

$$
\mathrm{S} \equiv\left[\phi\left(q^{*}(.), \omega, \beta, \sigma\right)-\phi(\tilde{q}, \omega, \beta, \sigma)\right]=\left[\left(q^{*}(.)-\tilde{q}\right)\left(v_{1}(\omega, \beta)-c_{1}(\omega, \sigma)\right)\right],
$$

est la différence entre le surplus joint $\phi\left(q^{*}(),. \omega, \beta, \sigma\right)$ résultant de la renégociation et le surplus joint généré par le contrat initial, c'est-à-dire $\phi(\widetilde{q}, \omega, \beta, \sigma)=\widetilde{q}\left[v_{1}(\omega, \sigma)-c_{1}(\omega, \sigma)\right]+\left[v_{2}(\omega, \sigma)-c_{2}(\omega, \sigma)\right]$. Suivant Edlin et Reichelstein [1996], nous supposons que le pouvoir de marchandage des deux parties est déterminé de manière exogène. Les parties se partagent alors le surplus; le vendeur recevant une fraction $\alpha \in[0,1]$ du surplus. Contrairement à la renégociation, nous ne modélisons par le processus de négociation lors de la formation du contrat initial. Nous supposons simplement que ces négociations aboutissent à un contrat efficace. Cela peut être obtenu en supposons que le vendeur choisit un contrat $(\tilde{q}, \tilde{p})$ sous la contrainte que l'acheteur atteint en moyenne son utilité de réservation, que nous normalisons à zéro.

Étant donné le contrat initial et la renégociation qui s'ensuit, le gain attendu de l'acheteur, lorsqu'il choisit un niveau d'investissement $\beta$ à la date 1 , s'écrit :

$$
\begin{aligned}
\mathrm{U}_{\mathrm{B}}(\beta, \sigma ; \tilde{q})=\int\left[\tilde{q} v_{1}(\omega, \beta)+v_{2}(\omega, \beta)-\tilde{p}\right]+(1-\alpha) \mathrm{S} d \mathrm{~F}(\omega)-\beta \\
=\int\left[\tilde{q} v_{1}(\omega, \beta)+v_{2}(\omega, \beta)-\widetilde{p}\right] \\
\quad+(1-\alpha)\left[\left(q^{*}(.)-\tilde{q}\right)\left(v_{1}(\omega, \beta)-c_{1}(\omega, \sigma)\right)\right] d \mathrm{~F}(w)-\beta
\end{aligned}
$$


où le premier terme de l'intégrale représente le gain de statu quo de l'acheteur avec le contrat initial. Le terme suivant représente la part que reçoit l'acheteur du surplus de la renégociation. De même, le gain attendu du vendeur de choisir un niveau d'investissement $\sigma$ à la date 1 s'écrit :

$$
\begin{aligned}
\mathrm{U}_{\mathrm{S}}(\beta, \sigma ; \tilde{q})=\int[ & {\left[\widetilde{p}-\widetilde{q} c_{1}(\omega, \sigma)+c_{2}(\omega, \sigma)\right]+\alpha \mathrm{S} d \mathrm{~F}(\omega)-\sigma } \\
=\int & \left.\int \widetilde{p}-\widetilde{q} c_{1}(\omega, \sigma)-c_{2}(\omega, \sigma)\right] \\
& \quad+\alpha\left[\left(q^{*}(.)-\widetilde{q}\right)\left(v_{1}(\omega, \beta)-c_{1}(\omega, \sigma)\right)\right] d \mathrm{~F}(w)-\sigma .
\end{aligned}
$$

L'acheteur choisit de façon non coopérative son investissement $\beta$ afin de maximiser $\mathrm{U}_{\mathrm{B}}(\beta, \sigma ; \widetilde{q})$. L'investissement d'équilibre, s'il est positif, satisfait la condition du premier ordre suivante :

$$
\begin{aligned}
\frac{\partial \mathrm{U}_{\mathrm{B}}(\beta, \sigma ; \tilde{q})}{\partial \beta}= & \alpha \tilde{q} \int v_{1}^{\prime}(\omega, \beta) d \mathrm{~F}(\omega)+(1-\alpha) \int v_{1}^{\prime}(\omega, \beta) q^{*}(.) d \mathrm{~F}(\omega) \\
& +\int v_{2}^{\prime}(\omega, \beta) d \mathrm{~F}(\omega)-1=0 .
\end{aligned}
$$

Le vendeur lui aussi choisit de façon non coopérative son investissement $\sigma$ pour maximiser $\mathrm{U}_{\mathrm{S}}(\beta, \sigma ; \tilde{q})$. Son investissement d'équilibre, s'il est positif, satisfait lui aussi la condition du premier ordre :

$$
\begin{aligned}
\frac{\partial \mathrm{U}_{\mathrm{S}}(\beta, \sigma ; \tilde{q})}{\partial \sigma}= & (1-\alpha) \widetilde{q} \int-c_{1}^{\prime}(\omega, \sigma) d \mathrm{~F}(\omega)+\alpha \int-c_{1}^{\prime}(\omega, \sigma) q^{*}(.) d \mathrm{~F}(\omega) \\
& -\int c_{2}^{\prime}{ }_{2}(\omega, \sigma) d \mathrm{~F}(\omega)-1=0 .
\end{aligned}
$$

Comme les investissements sont choisis simultanément, étant donné l'équation (4), $\beta^{*}$ est la meilleure réponse à $\sigma^{*}$ s'il existe une quantité initiale $\widetilde{q}_{\mathrm{B}}$ telle que :

$$
\frac{\partial \mathrm{U}_{\mathrm{B}}\left(\beta^{*}, \sigma^{*} ; \widetilde{q}_{\mathrm{B}}\right)}{\partial \beta}=\int v_{1}^{\prime}\left(\omega, \beta^{*}\right) q^{*}(.)+v_{2}^{\prime}\left(\omega, \beta^{*}\right) d \mathrm{~F}(\omega)=1 .
$$

De la même manière, étant donné l'équation (5), $\sigma^{*}$ constitue la meilleure réponse à $\beta^{*} \mathrm{~s}^{\prime}$ il existe une quantité initiale $\widetilde{q}_{\mathrm{S}}$ telle que :

$$
\frac{\partial \mathrm{U}_{\mathrm{S}}\left(\beta^{*}, \sigma^{*} ; \tilde{q}_{\mathrm{S}}\right)}{\partial \sigma}=\int-c_{1}^{\prime}\left(\omega, \sigma^{*}\right) q^{*}(.)-c_{2}^{\prime}\left(\omega, \sigma^{*}\right) d \mathrm{~F}(\omega)=1 .
$$

Nous obtenons alors la proposition suivante.

Proposition 1. Sous l'hypothèse 1, aucun contrat à obligation d'exécution susceptible d'être mis en œuvre par un juge ne peut résoudre le système d'equations (6-7) car les quantités optimales sont telles que $\widetilde{q}_{\mathrm{S}} \geqslant \int q^{*}(). d \mathrm{~F}(\omega)$ et $\tilde{q}_{\mathrm{B}} \geqslant \int q^{*}(). d \mathrm{~F}(\omega)$.

Preuve. En réécrivant l'équation (6), nous obtenons :

$$
\begin{aligned}
\frac{\partial \mathrm{U}_{\mathrm{B}}\left(\beta^{*}, \sigma^{*} ; \tilde{q}_{\mathrm{B}}\right)}{\partial \beta}= & \alpha \tilde{q}_{\mathrm{B}} \int v_{1}^{\prime}\left(\omega, \beta^{*}\right) d \mathrm{~F}(\omega)+(1-\alpha) \\
& \int v_{1}^{\prime}\left(\omega, \beta^{*}\right) q^{*}\left(\omega, \beta^{*}, \sigma^{*}\right) d \mathrm{~F}(\omega) \\
= & \int v_{1}^{\prime}\left(\omega, \beta^{*}\right) q^{*}\left(\omega, \beta^{*}, \sigma^{*}\right) d \mathrm{~F}(\omega)
\end{aligned}
$$


c'est-à-dire

$$
\tilde{q}_{\mathrm{B}}=\frac{\int v_{1}^{\prime}\left(\omega, \beta^{*}\right) q^{*}\left(\omega, \beta^{*}, \sigma^{*}\right) d \mathrm{~F}(\omega)}{\int v_{1}^{\prime}\left(\omega, \beta^{*}\right) d \mathrm{~F}(\omega)}
$$

comme $\int v_{1}^{\prime}\left(\omega, \beta^{*}\right) d \mathrm{~F}(\omega)>0$, nous avons alors

$$
\widetilde{q}_{\mathrm{B}} \geqslant \int q^{*}(\omega, .) d \mathrm{~F}(\omega)
$$

si la covariance suivante, ou inégalité algébrique de Tchebycheff (Wagener [2006])

$$
\int v_{1}^{\prime}\left(\omega, \beta^{*}\right) q^{*}\left(\omega, \beta^{*}, \sigma^{*}\right) d \mathrm{~F}(\omega) \geqslant \int v_{1}^{\prime}\left(\omega, \beta^{*}\right) d \mathrm{~F}(\omega) \int q^{*}(\omega, .) d \mathrm{~F}(\omega)
$$

est satisfaite. Comme $v_{1}^{\prime}(\omega,$.$) et q^{*}(\omega,$.$) sont des variables positives et crois-$ santes en $\omega$, d'après $v_{1}^{\prime}>0$ et $\partial v_{1} / \partial \beta \omega>0$ cette condition (11) est satisfaite. Du côté du vendeur, après réécriture de l'équation (7) et réarrangement des termes, nous obtenons :

$$
\tilde{q}_{\mathrm{S}}=\frac{\int-c_{1}^{\prime}\left(\omega, \sigma^{*}\right) q^{*}\left(\omega, \beta^{*}, \sigma^{*}\right) d \mathrm{~F}(\omega)}{\int-c_{1}^{\prime}\left(\omega, \sigma^{*}\right) d \mathrm{~F}(\omega)} .
$$

Comme $\int-c_{1}^{\prime}(\omega, \sigma) d \mathrm{~F}(\omega)>0$, nous obtenons alors :

$$
\tilde{q}_{\mathrm{S}} \geqslant \int q^{*}\left(\omega, \beta^{*}, \sigma^{*}\right) d \mathrm{~F}(\omega)
$$

si la condition suivante

$$
\int-c_{1}^{\prime}\left(\omega, \sigma^{*}\right) q^{*}(\omega, .) d \mathrm{~F}(\omega) \geqslant \int-c_{1}^{\prime}\left(\omega, \sigma^{*}\right) d \mathrm{~F}(\omega) \int q^{*}(\omega, .) d \mathrm{~F}(\omega)
$$

est satisfaite. Ce qui est le cas pour les mêmes raisons que précédemment.

Dans un environnement très général, le problème du hold-up ne peut donc être résolu en ayant recours à un simple contrat à obligation d'exécution. La proposition 1 ci-dessus montre que cela est dû au fait que les quantités optimales $\widetilde{q}_{\mathrm{S}}$ et $\widetilde{q}_{\mathrm{B}}$ qui doivent être spécifiées dans le contrat sont supérieures au niveau moyen d'échange attendu $\left(\int q^{*}(). d \mathrm{~F}(\omega)\right)$. Comme les fonctions de bénéfice et de coût sont dépendentes de l'aléa alors les équations (11) et (14) sont satisfaites parce que $\partial v_{1} / \partial \beta \omega>0$ et $\partial c_{1} / \partial \sigma \omega>0$. Ceci implique qu'un meilleur aléa augmente (diminue) le bénéfice (coût) marginal de l'investissement, et donc les quantités efficaces solution du système (6-7) doivent être augmentées par rapport au niveau moyen d'échange attendu afin d'inciter les parties à accroître leur niveau d'investissement. Comme les deux quantités sont différentes, puisque toutes les deux sont supérieures au niveau moyen d'échange, un contrat à obligation d'exécution spécifiant ces deux quantités ne peut être mis en œuvre par un juge. En effet, en cas de rupture du contrat le juge ne peut déterminer la partie qui a fait défaut, et donc il ne sait quelle quantité $\left(\widetilde{q}_{\mathrm{B}}\right.$ ou $\left.\widetilde{q}_{\mathrm{S}}\right)$ mettre en œuvre.

Ce résultat a deux conséquences. Dans un environnement très général, un contrat à obligation d'exécution ne peut être mis en œuvre par un juge que si l'on contraint le contrat initial en ajoutant la possibilité d'un contrôle du processus de renégociation (Aghion et al. [1994]). En effet, supposons que le contrat peut allouer l'ensemble du pouvoir de marchandage à l'une des parties, par exemple à l'acheteur $(\alpha=0)$. Dans ce cas, il vient immédiatement de l'équation (8) que $\widetilde{q}_{\mathrm{B}}=0$. Il ne reste alors plus qu'à choisir une quantité de défaut $\widetilde{q}=\widetilde{q}_{\mathrm{S}}$ 
satisfaisant l'équation (12) afin de résoudre le système d'équations (6-7). Le contrat optimal, ainsi défini, peut être mis en œuvre sans ambiguïté possible par un juge puisqu'un seul niveau d'échange par défaut est spécifié. Dans un environnement spécifique, Edlin et Reichelstein [1996] suggèrent que la solution efficace peut-être mise en œuvre sans ce contrôle contractuel du processus de renégociation, si les fonctions de bénéfice et de coût satisfont à l'hypothèse suivante de séparabilité.

HYPOTHÈSE 2. Les fonctions bénéfice et de coût sont telles que

$$
\begin{aligned}
& \mathrm{V}(q, \omega, \beta) \equiv v_{1}(\beta) q+v_{2}(q, \omega)+v_{3}(\omega, \beta) \\
& \mathrm{C}(q, \omega, \sigma) \equiv c_{1}(\sigma) q+c_{2}(q, \omega)+c_{3}(\omega, \beta) .
\end{aligned}
$$

Cette hypothèse implique une hypothèse de séparabilité bien plus forte que celle de l'hypothèse 1, puisque les fonctions sont aussi additivement séparables en l'aléa et en l'investissement. Considérons maintenant l'hypothèse suivante.

HYPOTHÈSE 3. $\mathrm{V}(\omega, \beta ; q) \equiv \mathrm{V}(0, \beta ; q)=v_{1}(\beta) q+v_{2}(\beta)$ et $\mathrm{C}(\omega, \beta ; q) \equiv \mathrm{C}(0, \sigma ; q)=c_{1}(\sigma) q+c_{2}(\sigma)$ sont indépendentes de l'aléa $\omega$.

Cette hypothèse dit simplement que les fonctions de bénéfice et de coût sont indépendantes de l'aléa. Autrement dit, il n'y a pas d'incertitude directe dans les fonctions de bénéfice et de coût de production. L'incertitude provient seulement de la quantité efficace qui sera échangée ex post. La proposition 2 ci-dessous montre que l'hypothèse 2 est équivalente à l'hypothèse 3 , car ces deux hypothèses impliquent que le biais mis en évidence dans la proposition 1 disparaît. Les deux quantités efficaces sont alors égales au niveau moyen de l'échange qui sera réalisé ex post, ce qui permet à un contrat à obligation d'exécution de mettre en œuvre la solution efficace.

Proposition 2. Sous l'hypothèse 2 (i) ou l'hypothèse 3 (ii), les quantités initiales ne sont plus biaisées puisque $\tilde{q}=\widetilde{q}_{\mathrm{B}}=\widetilde{q}_{\mathrm{S}}=\int q^{*}(). d \mathrm{~F}(\omega)$. Un contrat à obligation d'exécution $(\widetilde{q}, \widetilde{p})$ peut alors implémenter la solution efficace.

Preuve. (i) Sous l'hypothèse 2, en réécrivant (6) nous obtenons :

$$
\begin{aligned}
\frac{\partial \mathrm{U}_{\mathrm{B}}\left(\beta^{*}, \sigma^{*} ; \widetilde{q}_{\mathrm{B}}\right)}{\partial \beta} & =(1-\alpha) v_{1}^{\prime}\left(\beta^{*}\right) \int q^{*}\left(\omega, \beta^{*}, \sigma^{*}\right) d \mathrm{~F}(\omega)+\alpha v_{1}^{\prime}\left(\beta^{*}\right) \widetilde{q}_{\mathrm{B}} \\
& =v_{1}^{\prime}(\beta) \int q^{*}\left(\omega, \beta^{*}, \sigma^{*}\right) d \mathrm{~F}(\omega)
\end{aligned}
$$

ce qui implique :

$$
\tilde{q}_{\mathrm{B}}=\frac{\alpha v_{1}^{\prime}\left(\beta^{*}\right) \int q^{*}\left(\omega, \beta^{*}, \sigma^{*}\right) d \mathrm{~F}(\omega)}{\alpha v_{1}^{\prime}\left(\beta^{*}\right)}
$$

ou $\widetilde{q}_{\mathrm{B}}=\int q^{*}(). d \mathrm{~F}(\omega)$. De façon similaire, en réécrivant (7) nous obtenons

$$
\begin{aligned}
\frac{\partial \mathrm{U}_{\mathrm{S}}\left(\beta^{*}, \sigma^{*} ; \tilde{q}_{\mathrm{S}}\right)}{\partial \sigma} & =-\alpha c_{1}^{\prime}(\sigma) \int q^{*}\left(\omega, \beta^{*}, \sigma^{*}\right) d \mathrm{~F}(\omega)+(1-\alpha) c_{1}^{\prime}(\sigma) \widetilde{q}_{\mathrm{S}} \\
& =-c_{1}^{\prime}(\sigma) \int q^{*}\left(\omega, \beta^{*}, \sigma^{*}\right) d \mathrm{~F}(\omega)
\end{aligned}
$$


soit

$$
\tilde{q}_{\mathrm{S}}=\frac{-(1-\alpha) c_{\sigma}^{\prime}\left(\sigma^{*}\right) \int q^{*}\left(\omega, \beta^{*}, \sigma\right) d \mathrm{~F}(\omega)}{-(1-\alpha) c_{\sigma}^{\prime}\left(\sigma^{*}\right)}
$$

autrement dit, $\tilde{q}_{\mathrm{S}}=\int q^{*}(). d \mathrm{~F}(\omega)$. De ce fait, $\tilde{q}=\tilde{q}_{\mathrm{B}}=\tilde{q}_{\mathrm{S}}=\int q^{*}(). d \mathrm{~F}(\omega)$. Une quantité unique $\widetilde{q}$ peut donc résoudre le système d'équations (6-7).

(ii) Sous 1'hypothèse 3, en réécrivant (6), on obtient (17) et donc (18). De façon similaire, en réécrivant (7), nous obtenons (19) et donc (20). Ceci implique comme précédemment que $\tilde{q}=\widetilde{q}_{\mathrm{B}}=\widetilde{q}_{\mathrm{S}}=\int q^{*}(). d \mathrm{~F}(\omega)$.

Pour expliquer le résultat d'équivalence de la proposition 2, remarquons que pour générer la solution efficace ( $c f$. équations (17) et (19)), ce qui est crucial c'est le fait que les premiers termes, $v_{1}(\beta) q$ et $c_{1}(\sigma) q$, des fonctions de bénéfice et de coût dans l'hypothèse 2 sont indépendants de l'aléa $\omega$. Ce qui correspond à l'hypothèse 3. Ce constat est confirmé par l'interprétation économique de cette hypothèse 2 . En effet, un euro d'investissement spécifique réalisé par un acheteur (vendeur) augmente (diminue) son bénéfice (coût de production) unitaire de $v_{1}^{\prime}(\beta)\left(c_{1}^{\prime}(\sigma)\right)$. L'impact de l'investissement est donc bien indépendant de l'aléa (Edlin et Reichelstein [1995], p. 281). L'absence d'effet de l'aléa sur le rendement marginal de l'investissement fait donc disparaître le biais sur les quantités optimales, qui seront alors fixées au niveau moyen d'échange ex post $\left(\widetilde{q}_{\mathrm{B}}=\widetilde{q}_{\mathrm{S}}=\int q^{*}(). d \mathrm{~F}(\omega)\right)$. Ceci implique alors que l'on peut définir un contrat optimale stipulant une quantité par défaut unique, et donc exécutable par un juge. Ce résultat d'équivalence a une conséquence importante, puisque nous montrons ci-dessous que, sous l'hypothèse 3 , un contrat volontaire met en œuvre de façon triviale la solution efficace.

\section{L'efficacité en contrat volontaire}

Supposons maintenant que les parties signent à la date 0 , non pas un contrat à obligation d'exécution mais un contrat volontaire. Ce contrat spécifie un menu de deux prix $p(q)$, avec $p(q)=p_{1}$ si $q=1$ et $p(q)=p_{0}$ si $q=0$; ce prix devant être payé par l'acheteur au vendeur lorsqu'une quantité $q$ est échangée (Hart et Moore [1988]). Contrairement au contrat d'exécution forcée, aucun niveau d'échange minimal $\tilde{q}$ n'est spécifié. La proposition 3 ci-dessous montrent que ce type de contrat permet d'atteindre la solution efficace.

Proposition 3. Sous l'hypothèse 3, un contrat volontaire $\left(p_{1}, p_{0}\right)$ peut mettre en auvre la solution efficace.

Preuve. Lorsqu'il n'y a aucun effet direct de l'aléa sur les fonctions de bénéfice et de coût, les parties peuvent soit échanger la quantité efficace $q^{*}()=$.1 , soit ne rien échanger $\left(q^{*}()=0.\right)$. Pour atteindre la solution efficace, le contrat initial doit donc simplement partager le surplus de façon à ce que les parties souhaitent échanger. Choisissons alors le contrat $\left(p_{1}, p_{0}\right)$ tel qu'à la date 2 , $v_{1}(\beta) q^{*}()+.v_{2}(\beta) \geqslant p_{1}-p_{0} \geqslant c_{1}(\sigma) q^{*}()+.c_{2}(\sigma)$. De ce fait, l'échange apparaît au prix $p_{1}$ sans renégociation du contrat initial car l'échange est efficace 
et aucune partie n'a intérêt à bloquer la réalisation de l'échange. L'utilité anticipée de l'acheteur s'écrit alors $\mathrm{U}_{\mathrm{B}}\left(\beta, p_{1}\right)=v_{1}(\beta) \int q^{*}(). d \mathrm{~F}(\omega)+v_{2}(\beta)-p_{1}$ et celle du vendeur s'écrit $\mathrm{U}_{\mathrm{S}}\left(\sigma, p_{1}\right)=p_{1}-c_{1}(\sigma) \int q^{*}(). d \mathrm{~F}(\omega)-c_{2}(\sigma)$. En différenciant, on obtient :

$$
\frac{\partial \mathrm{U}_{\mathrm{B}}\left(\beta^{*}\right)}{\partial \beta}=v_{1}^{\prime}\left(\beta^{*}\right) \int q^{*}(.) d \mathrm{~F}(\omega)+v_{2}^{\prime}\left(\beta^{*}\right)=1
$$

et

$$
\frac{\partial \mathrm{U}_{\mathrm{S}}\left(\sigma^{*}\right)}{\partial \sigma}=-c_{1}^{\prime}\left(\sigma^{*}\right) \int q^{*}(.) d \mathrm{~F}(\omega)-c_{2}^{\prime}\left(\sigma^{*}\right)=1
$$

respectivement. Ceci implique que le système d'équations (6-7) est trivialement satisfait sous l'hypothèse 3 , car leurs incitations à investir sont alignées sur le rendement social des investissements. De ce fait, la solution de premier rang $\left(\beta^{*}, \sigma^{*}\right)$ peut être atteinte avec un contrat volontaire $\left(p_{1}, p_{0}\right)$.

\section{CONCLUSION}

Cet article montre qu'un contrat à obligation d'exécution ne peut résoudre le problème du hold-up que sous une condition restrictive, à savoir la possibilité de contrôler contractuellement le processus de renégociation. Ce résultat permet d'expliquer la faible utilisation dans les pays de droit civil, comme dans ceux de Common Law, de ce remède à la rupture des contrats qu'est l'obligation d'exécution (Lando et Rose [2004]). En outre, il rend compte de l'utilisation de ce remède dans des contrats « complexes », comme les contrats de construction où des mécanismes de pénalités à payer en cas d'inexécution existent; ce qui permet de contrôler le processus de marchandage si une renégociation survient (Shavell [2006]).

\section{RÉFÉRENCES BIBLIOGRAPHIQUES}

AGHion P., DewATRIPONT M., REY P. [1994], « Renegotiation design with unverifiable information », Econometrica, 62 (2), p. 257-282.

Al-NajJar N., Anderlini L., Felli L. [2006], «Undescribable events », Review of Economic Studies, 73, p. 849-868.

ANDERLINI L., FeLLI L. [1994], « Incomplete Written Contracts: Indescribable States of Natur », Quarterly Journal of Economics, 109, p. 1085-1124.

Anderlini L., Felli L., Postlewaite A. [2007], « Courts of law and unforseen contingencies », Journal of Law, Economics and Organization, 23, p. 662-684.

Battigalli P., MAGGi G. [2002], «Rigidity, discretion, and the costs of writing contracts », American Economic Review, 92 (4), p. 798-817.

DYE R. [1985], « Costly contracts contingencies », International Economic Review, 26, p. 233-250.

EDLIN A., REICHELSTEIN S. [1995], «Specific investment under negotiated transfer pricing: An efficiency result », Accounting Review, 70 (2), p. 275-291. 
EDLIN A., REICHELSTEIN S. [1996], « Holdups, standard breach remedies, and optimal investment », American Economic Review, 86, p. 478-501.

Grossman S., HART O. [1986], "The costs and benefits of ownership: A theory of vertical and lateral integration », Journal of Political Economy, 94 (4), p. 691-719.

HART O. [1995], Firms, Contracts, and Financial Structure, Oxford University Press, Oxford.

HART O., MOORE J. [1988], « Incomplete contracts and renegotiation », Econometrica, 56, p. 755-786.

KLEIN B., CRAWFord C., AlChiAn A. [1978], « Vertical integration, appropriable rents and the competitive contracting process », Journal of Law and Economics, 21, p. 297326.

LANDO H., RosE C. [2004], « On the enforcement of specific performance in civil law countries ", International Review of Law and Economics, 24, p. 473-487.

MACLEOD B., MALCOMSON J. [1993], « Investments, hold-up, and the form of market contracts ", American Economics Review, 83, p. 811-837.

NoldEKE G., SCHMIDT K. [1995], « Option contracts and renegotiation: a solution to the hold-up problem », Rand Journal of Economics, 26 (2), p. 163-179.

SHAVELL S. [2006], « Specific performance versus damages for breach of contract: An economic analysis », Texas Law Review, 84, p. 831-875.

WAGENER A. [2006], « Chebyshev's algebraic inequality and comparative statics under uncertainty », Mathematical Social Sciences, 52, p. 217-221.

WiLliamson O. [1985], The Economic Institutions of Capitalism, New York, The Free Press. 
